\title{
Osteo/odontogenic Differentiation of Human Mesenchymal Stem Cells with Platelet-rich Plasma and Mineral Trioxide
}

\section{Aggregate}

\author{
Amit Vanka ${ }^{1}$, Sandeep Kumar Vishwakarma ${ }^{2}$, Manohar K Bhat ${ }^{3}$, Shanthi Vanka ${ }^{4}$, Othman Wali ${ }^{5}$, Aleem A Khan $^{6}$
}

\begin{abstract}
Aim: Aim of the study was to investigate the effect of PRP and MTA individually and combined on in vitro human bone marrow mesenchymal stem cells' (MSCs) proliferation and osteo/odontogenic differentiation potential.

Materials and methods: MSCs were cultured in vitro with MTA, 5\% PRP, 10\% PRP, MTA with 5\%PRP, and MTA with 10\% PRP. Fetal calf serum (FCS) was used as control. Cell viability and proliferative efficiency were tested with cell adhesion and MTT assay. Osteo/odontogenic differentiation was assessed and quantified with alizarin red staining.

Results: MTA alone, MTA with 5\% PRP, and MTA with 10\% PRP showed significantly high proliferation at day 7 and 14 when compared to the control group. Enhanced differentiation and the highest calcium deposition was observed in MTA with the $10 \%$ PRP group.

Conclusion: Within limitations of the in vitro environment, results imply an increased proliferation and induction of MSCs into osteo/odontogenic differentiation by the combination rather than a mere sealing of PRP by MTA.

Clinical significance: PRP and MTA have the potential for true regeneration of the pulp tissue. Moreover, the combination of PRP and MTA can be utilized to expand the MSCs to generate adequate numbers for clinical applications, without xenogenic contamination.

Keywords: Laboratory research, Mineral trioxide aggregate, Platelet-rich plasma, Stem cells.

The Journal of Contemporary Dental Practice (2019): 10.5005/jp-journals-10024-2677
\end{abstract}

\section{INTRODUCTION}

Management of immature pulpless teeth is challenging and recent research has been focused on finding alternatives to conventional endodontic procedures. Regenerative endodontics can potentially replace damaged structures, including dentin and cells of the pulp-dentin complex. Regenerative techniques have focused primarily on either root canal revascularization via blood clotting or postnatal stem cell therapy. ${ }^{1}$ Case reports and series published on revascularization of pulp for immature pulpless teeth with induced blood-clot have shown encouraging results. ${ }^{2,3}$ Nevertheless, certain areas of concern remain unaddressed. One of the crucial questions to be resolved during regeneration is the phenotype of the tissue formed. ${ }^{4}$ Most reports concur that the tissue formed with a blood clot is an extension of the periodontal ligament and is associated with cementum deposition. ${ }^{5}$ Since the concentration and composition of cells trapped in the fibrin clot cannot be controlled, blood clot formation cannot constitute tissue engineering in the true sense ${ }^{1}$ and alternatives need to be explored.

Tissue engineering is based on a combination of stem cells, scaffolds, and growth factors. Post-natal human mesenchymal stem cells (MSCs) have the capacity to self-renew and retain sufficient proliferative and differentiation potential for reconstitution of specific tissues. ${ }^{6}$ One of the major limitations in the use of MSCs is their limited availability from primary source of tissues, given that clinical applications require a significantly high number of cells to achieve a successful result. ${ }^{7}$ Xenogenic supplements rich in growth factors, such as fetal calf serum (FCS), are currently used for expanding and differentiating MSCs, but concerns regarding immunogenicity and internalization of xenogenic proteins ${ }^{8}$ render them unsuitable for clinical applications.

\begin{abstract}
${ }^{1}$ Faculty of Dental Science, Pacific Academy of Higher Education and Research University, Udaipur, Rajasthan, India

${ }^{2,6}$ Centre for Liver Research and Diagnostics (CLRD), Central Laboratory for Stem Cell Research and Translational Medicine, Deccan College of Medical Sciences, Hyderabad, Telangana, India

${ }^{3}$ Department of Pedodontics and Preventive Dentistry, Jaipur Dental College, Jaipur, Rajasthan, India

${ }^{4}$ Department of Preventive Dental Sciences, Ibn Sina National College for Medical Studies, Jeddah, Kingdom of Saudi Arabia

${ }^{5}$ Ibn Sina National College for Medical Studies, Jeddah, Kingdom of Saudi Arabia

Corresponding Author: Amit Vanka, Faculty of Dental Science, Pacific Academy of Higher Education and Research University, Udaipur, Rajasthan, India, Phone: +966 597697765, e-mail: amitvanka18@ gmail.com

How to cite this article:Vanka A, Vishwakarma SK, Bhat MK, et al. Osteo/ odontogenic Differentiation of Human Mesenchymal Stem Cells with Platelet-rich Plasma and Mineral Trioxide Aggregate. J Contemp Dent Pract 2019;20(10):1171-1178.
\end{abstract}

Source of support: Nil

Conflict of interest: None

Platelet-rich plasma (PRP) is unique wherein it is an autologous source of growth factors and can serve as a scaffold to carry cells. ${ }^{9}$ PRP has been proposed for ex vivo expansion of MSCs from different sources. ${ }^{10,11}$ A small but growing number of clinical case reports demonstrate that revascularization of an endodontically compromised immature tooth is possible with PRP. ${ }^{12,13}$ Pulp-like tissue can be generated in a human tooth with a previous necrotic pulp and open apex using PRP alone without blood clot. ${ }^{14}$ 
Clinically, PRP is sealed into the canal with mineral trioxide aggregate (MTA) as part of the revascularization procedure..$^{12-14}$ The sealing ability of MTA is well documented ${ }^{15}$ as its biocompatibility ${ }^{16}$ and proliferative effect on MSCs. ${ }^{17}$ However, their combined effect on the mesenchymal stem cell population and potentially on the eventual tissue regenerated has not yet been explored. Hence, in the present study, we investigated the effects of PRP in the presence of MTA on MSCs' proliferation and osteo/ odontogenic differentiation potential.

\section{Materials and Methods}

The study was conducted after taking approval from institutional review board. Signed informed consent forms were collected from each volunteer enrolled in the study.

\section{Preparation of PRP}

Whole blood collected from 10 healthy volunteers with no relevant diseases and free of any drugs known to affect platelet functions was acquired from the blood bank. A double centrifugation method was used for PRP preparation modified from a previous study. ${ }^{18}$ Briefly, the first centrifugation (soft spin) was carried out at $130 \times g$ for 15 minutes at room temperature. Three fractions were observed after centrifugation containing a lower dense layer containing red blood cells, a thin white intermediate layer with leukocytes and platelets (buffy coat), and the upper yellow fraction. The first clouded phase containing platelets, platelet-poor plasma (PPP), and buffy coat was collected directly by gentle aspiration using a micropipette. Further PRP was concentrated using a second 15 minutes centrifugation step at $280 \times g$. Supernatant was partially discarded and the PRP pellet was resuspended in plasma to obtain PRP. The concentration of the platelets within the PRP was assessed, and standardized to $1200 \times 10^{3}$ platelets $/ \mathrm{mL}$ by adding the appropriate amount of PPP.

\section{Isolation and Enrichment of MSCs}

MSCs were isolated from human bone marrow blood as described elsewhere ${ }^{19}$ and cultured at $37^{\circ} \mathrm{C}$ temperature and $5 \% \mathrm{CO}_{2}$ atmosphere in sterile tissue culture plates (Corning, USA). Wherever required, separate cohort studies were carried out to validate the experimental results. Isolated bone marrow cells were seeded in triplicate cultures (six-well plates) at two different cell densities $\left(1 \times 10^{6}\right.$ cells/well and $2 \times 10^{6}$ cells/well). Complete human Mesencult proliferation medium supplemented with stimulatory factors (StemCell Technologies, Canada) were added to each well. The frequency of colony formation was evaluated by colony forming unit-fibroblast (CFU-F) after 14 days of culture. Cultured cells were washed twice with calcium and magnesium free phosphate buffer saline (PBS; Cat\#:P3813, Sigma, USA) and fixed in cold ethanol. CFU-Fs were stained with Giemsa stain (Cat\#:G5637, SIGMA, USA) and colonies with $>50$ cells were identified and counted under the light microscopy.

Similar protocol was applied for CFU-OB assay wherein osteogenic stimulatory supplements (StemCell Technologies, Canada) were used for osteo/odontogenic differentiation of MSCs. Cell densities for CFU-OB assay was maintained similar to the CFU-F assay $\left(1 \times 10^{6}\right.$ cells/well and $2 \times 10^{6}$ cells/well respectively). The frequency of osteogenic colony formation was identified at day 14 post-stimulation following staining with alkaline phosphatase (Cat\#: APF-1KT, Sigma, USA) and 1.0\% methylene blue (Cat\#: M9140, Sigma, USA) prepared in borate buffer. The frequency of formation of osteo/odontogenic colonies was observed under light microscope.

\section{Mixing of ProRoot White MTA}

MTA was mixed as per manufacturer's instructions (DENTSPLY, Endodontics). Briefly, $100 \mathrm{mg}$ of ProRoot white MTA was mixed with $35 \mu \mathrm{L}$ of sterile water under a laminar flow hood in a sterile container for approximately 1 minute. The mix was then coated onto the surface of the cell culture plates using a sterile applicator brush to achieve a homogeneous coating. MTA was then incubated for 24 hours at $37^{\circ} \mathrm{C}$ in $5 \% \mathrm{CO}_{2}$ and $100 \%$ humidity before cell seeding.

\section{Seeding of Cells}

MSCs were seeded in cell culture plates coated with MTA. $10 \%$ FCS in proliferation medium was substituted with $5 \%$ and $10 \%$ PRP, respectively. To wells coated with MTA, 5\% and 10\% PRP thus prepared was also added to overall constitute five test groups. The control group consisted of cell suspensions added to proliferation medium containing $10 \%$ FCS without MTA coating. Cell suspensions were seeded for all the groups in triplicate on 96 well flat bottomed plate (Fig. 1). All concentrations of PRP were activated using $10 \%$ calcium gluconate (Cat\#:227641, Sigma, USA).

\section{Cell Adhesion Assay}

A total of $2 \times 10^{4}$ cells were seeded on cell culture plates directly for PRP or control and on MTA coated surfaces for MTA and with PRP groups. Cells were incubated for 72 hours at $37^{\circ} \mathrm{C}$ and $5 \%$ $\mathrm{CO}_{2}$ atmosphere for initial adherence. Following incubation, nonadherent cells were gently washed with $1 \times$ PBS and adherent cells were stained with fluorescein diacetate (FDA) $(1 \mathrm{mg} / \mathrm{mL}$, Cat\#: F7378-5G, Sigma, USA). Cell were incubated for 15 minutes at $37^{\circ} \mathrm{C}$, washed twice with $1 \times$ PBS and observed under a fluorescence microscope (Axiovert 200M, Germany) to identify the cell membrane integrity and viability.

\section{MTT Cell Proliferation Assay}

3-(4,5-Dimethylthiazol-2-yl)-2,5-diphenyltetrazolium bromide (MTT, Cat\#: TC191-1G, Himedia, India) cell proliferation assay was performed at day 3, 7, and 14 to assess MSCs proliferation efficiency. Briefly, $1.2 \times 10^{4}$ cells were seeded in each well of 96 well cell culture plates in different groups as described above. Cells were allowed to proliferate for 14 days and assessed for MTT reduction at different time periods. Formazan crystals were dissolved using acidified isopropanol solution. The absorbance was measured at $570 \mathrm{~nm}$ using a microplate reader (Bio-Rad, USA) and compared in each group.

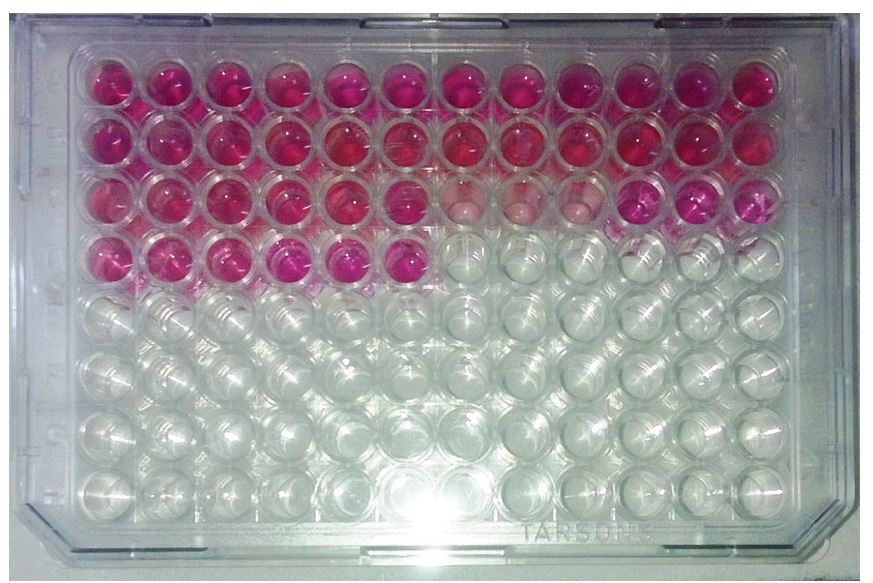

Fig. 1: Cell suspensions at a concentration of $1.2 \times 10^{4}$ cells/well were seeded for control and experimental groups in triplicate on $96-$ well flat bottomed plate 


\section{Osteo/odontogenic Differentiation Potential of MSCs Cultured in Different Conditions}

MSCs derived from Dulbecco's modified eagle's medium (DMEM, Cat\#: AT068, Himedia, India) supplemented with 5\% PRP, 10\% PRP, 5\% PRP with MTA and 10\% PRP with MTA, were induced to osteo/ odontogenic differentiation using osteogenic differentiation supplements (MesenCult ${ }^{\text {TM }}$ XF osteogenic stimulatory kit, human, Cat\#: 05434, Stem Cell Technologies, Canada). MSCs derived from DMEM with $10 \%$ FCS were used as control. Cells were allowed to differentiate for 14 days and characterized for their differentiation potential.

\section{Alizarin Red Staining}

After 14 days, differentiated cells from each group were washed thrice with $1 \mathrm{X}$ PBS and then stained with $10 \%$ alizarin red solution (Cat\#: 40-1009-5-500ML-J, Sigma, USA) at room temperature to assess the mineralization and presence of calcium deposits. Cells were again washed twice with $1 \times$ PBS to remove excess staining from the culture plates. To quantify the alizarin red staining, $1 \mathrm{~mL}$ of $10 \%$ cetylpyridium chloride (Cat\#: C0732, Sigma, USA) was added to each well and incubated for 20 minutes at room temperature. An estimated $100 \mu \mathrm{L}$ of eluted stain was transferred to 96 well plates from each group in triplicate. Absorbance was measured at $550 \mathrm{~nm}$ using a spectrophotometer. A standard curve was created using cetylpyridium chloride and alizarin red stain and the known samples were quantified by plotting absorbance values of samples on standard plots. Calcium concentration was quantified with colorimetric assay.

\section{Statistical Analysis}

The results were expressed as mean and standard deviation. Statistical test used to compare means was performed using two-way analysis of variance (ANOVA) and Tukey post hoc with GraphPad Prism software (Version 5). The $p$ value $<0.01$ was considered to be statistically significant.

\section{Results}

\section{Frequency of Colony Formation}

Microscopic observation during the estimation of CFU-F for MSCs and osteo/odontogenic cells derived from MSCs at day 14 revealed

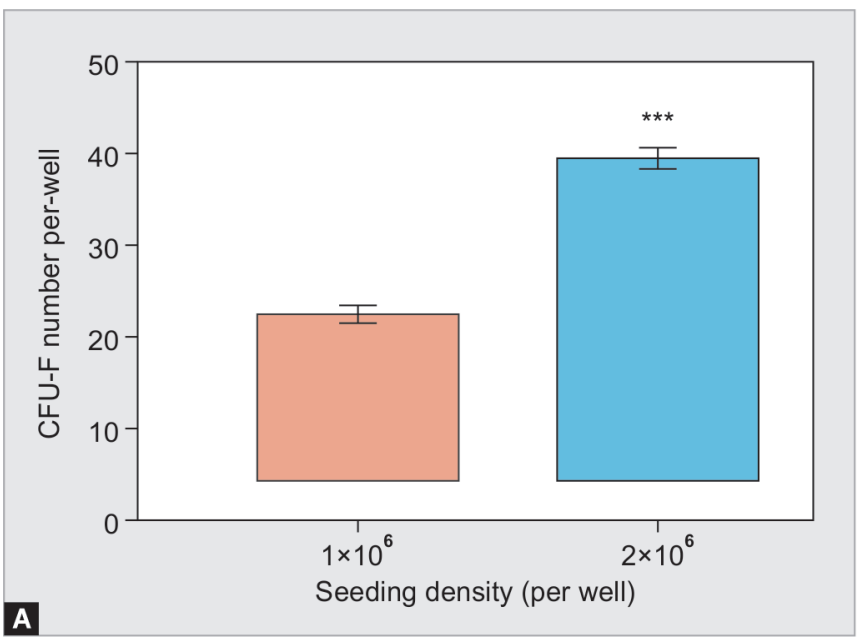

an enhanced frequency for colony formation in both conditions. Higher cell seeding density $\left(2 \times 10^{6}\right.$ cells/well) was able to develop an increased number of colonies when compared to less seeding density $\left(1 \times 10^{6}\right.$ cells/well) $\left.{ }^{* *} p<0.001\right)$ for both CFU-F (Fig. $\left.2 \mathrm{~A}\right)$ and CFU-OB (Fig. 2B).

\section{Relative Cell Viability and Growth Rate}

Phase contrast and FDA fluorescence microscopic images of cultured MSCs at day 3 showed significant adherence and change in morphology from spherical to spindle shape (Fig. 3A). MSCs exhibited significantly enhanced cell adhesion in MTA $\left({ }^{* * *} p<0.0001\right), 10 \% \operatorname{PRP}\left({ }^{*} p<0.01\right)$, MTA with $5 \% \operatorname{PRP}\left({ }^{* * *} p<0.0001\right)$ and MTA with $10 \%$ PRP $\left({ }^{* *} p<0.0001\right)$ groups when compared to control (Fig. 3B). Further quantification of cellular viability and proliferation using MTT assay at day 3, 7, and 14 revealed no significant difference between control, 5\% PRP and 10\% PRP at all time points (Tables $1 \mathrm{~A}$ and B). MTA alone, MTA with 5\% PRP, and MTA with $10 \%$ PRP showed significantly high proliferation at day 7 and 14 when compared with the control group.

\section{Induced Mineralization During Osteo/Odontogenic Differentiation}

Increased differentiation was observed (Tables 2A and B) and alizarin red staining showed prominent positive staining in differentiated cells, at 14 days in each group. However, it was highest in MTA with 10\% PRP (Fig. 4A). This observation was further evaluated quantitatively by estimating calcium deposition in differentiated cells (Fig. 4B). The highest calcium deposition was again observed in MTA with 10\% PRP group when compared to the control $\left({ }^{* *} p<0.001\right)$.

\section{Discussion}

PRP has been used in a host of clinical applications, including wound healing after surgery, sports injuries, and tissue regeneration. Platelet activation is the basis of PRP action. However, the biological characteristics of PRP rely on the concentration of platelets and the biological activity of PRP differs according to the preparation technique. Standardized preparations can help PRP secrete many growth factors (GFs) at high concentrations, including transforming growth factor- $\beta$, platelet-derived growth factor, insulin-like growth factor, vascular

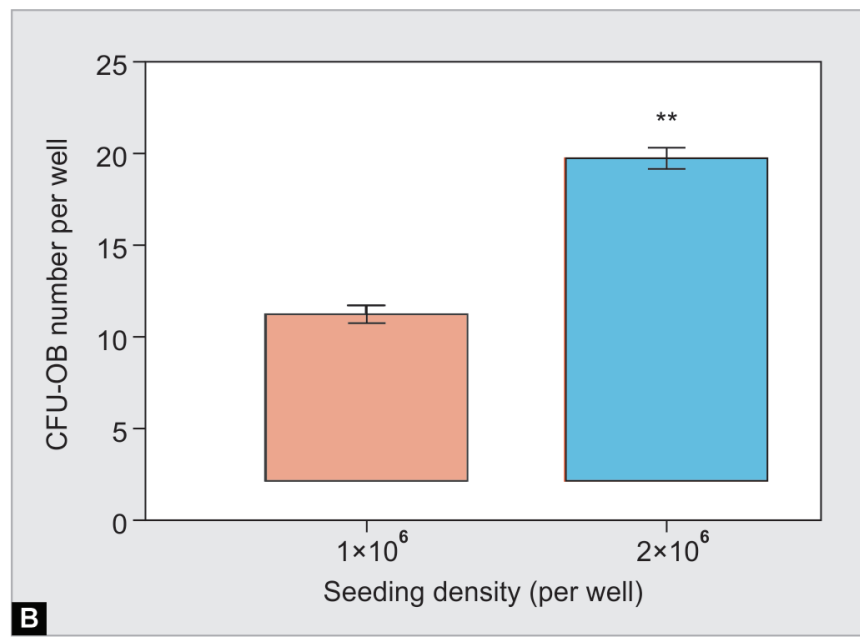

Figs $2 \mathrm{~A}$ and B: CFU-F and CFU-OB assay revealed enhanced colony formation at day 14 for MSCs (A) and osteo/odontogenic cells (B) during in vitro culture in 6 well plates 


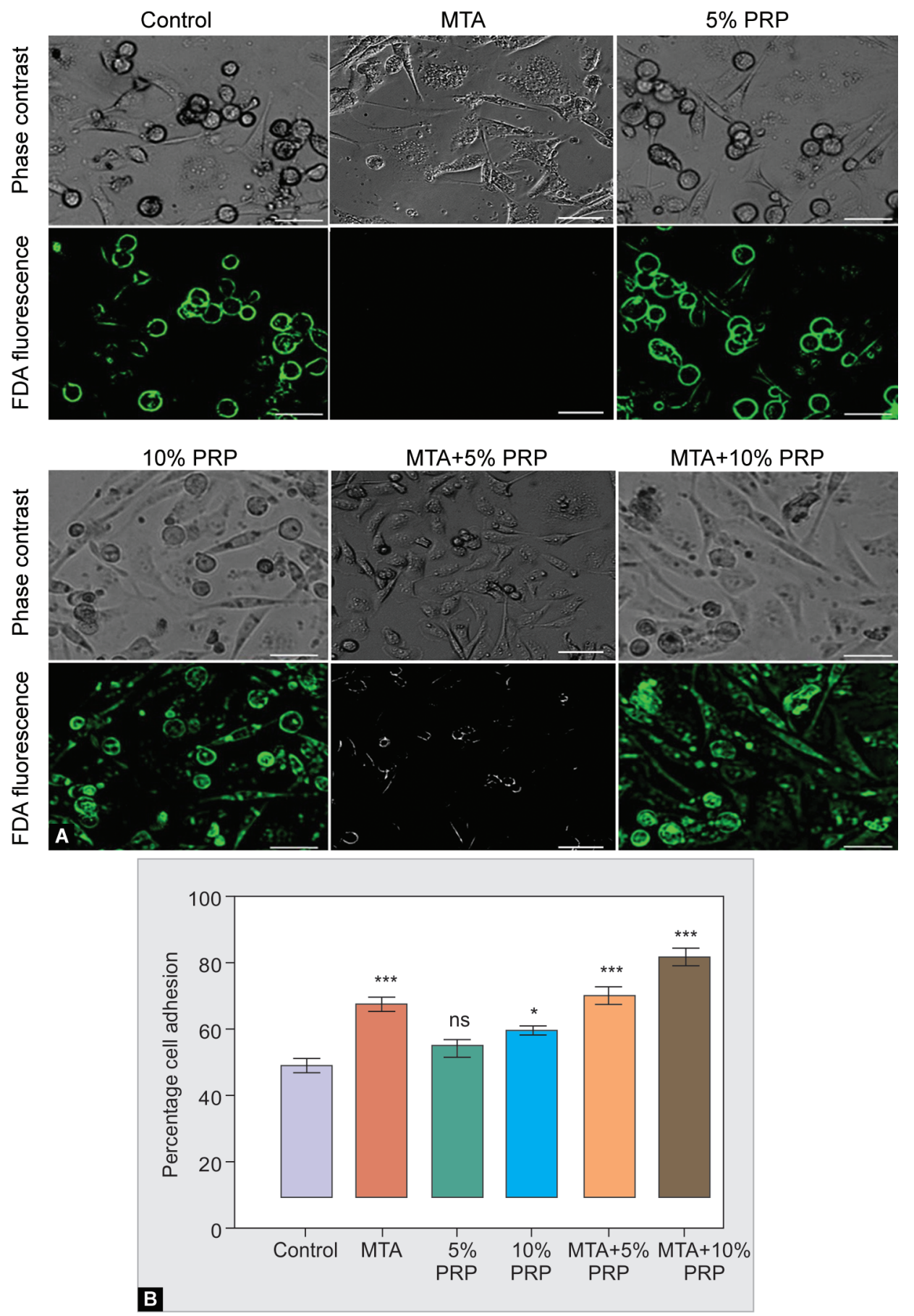

Figs 3A and B: (A) FDA fluorescence microscopic images of cultured MSCs at day 3 showing significant adherence and change in morphology from spherical to spindle shape; (B) Percentage cell adhesion determined by the fluorescent intensity of FDA was significantly high in MTA, 10\% PRP, MTA + 5\% PRP, and MTA + 10\% PRP. Cell adhesion was highest in MTA + 10\% PRP group

endothelial growth factor, and epidermal growth factor. ${ }^{20}$ The technique used in our study yielded 1.2 million $/ \mathrm{mL}$ concentration of platelets in PRP, which closely corresponds to working definition for therapeutic PRP of 1 million $/ \mathrm{mL}$ platelet count. ${ }^{21}$ This count also falls in the range of 3-6 fold enhancement that is deemed necessary to demonstrate clinical efficacy. ${ }^{22}$ The preparation contained both platelets and leukocytes and can be classified as leukocyte-rich PRP (L-PRP). ${ }^{23}$ Greater platelet recovery and efficacy have been reported with L-PRP ${ }^{24}$ and was hence selected for the current study. Moreover it is in this family that the largest number of commercial or experimental systems exist. ${ }^{21}$

MSCs are extensively studied cell types in regenerative medicine owing to their immunomodulatory properties. Bonemarrow-derived stem cells are the most frequently investigated cell type, best characterized, and often designated as the gold standard. ${ }^{25,26}$ While other sources of MSCs, particularly from various 


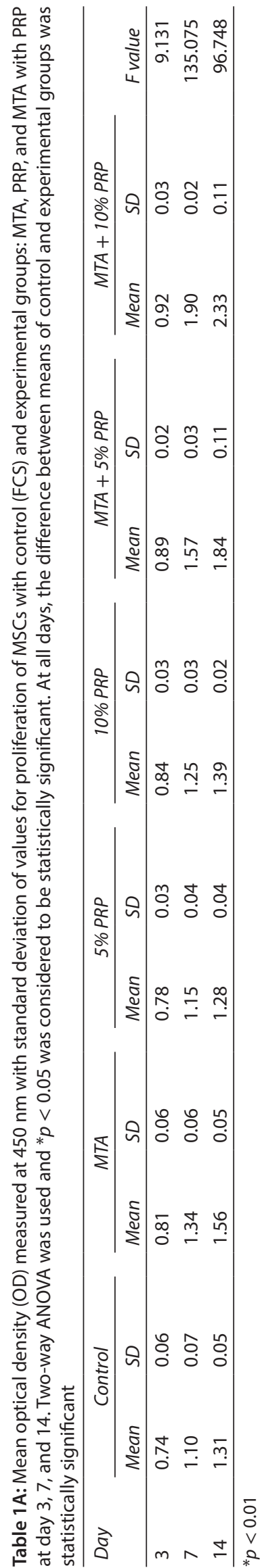

Table 1B: Multiple comparison by Tukey post hoc showed a significant increase in absorbance in MTA alone and MTA with 5\% and 10\% PRP respectively at day 7 and 14 . No significant difference was observed between control, 5\% PRP and 10\% PRP at day 14

\begin{tabular}{llll}
\hline Multiple comparsions (MSC proliferation) & Day 3 & Day 7 & Day 14 \\
\hline Control vs MTA & No & Yes & Yes \\
Control vs 5\% PRP & No & No & No \\
Control vs $10 \%$ PRP & No & Yes & No \\
Control vs MTA + 5\% PRP & Yes & Yes & Yes \\
Control vs MTA + 10\% PRP & Yes & Yes & Yes \\
MTA vs 5\% PRP & No & Yes & Yes \\
MTA vs 10\% PRP & No & No & No \\
MTA vs MTA + 5\% PRP & No & Yes & Yes \\
MTA vs MTA + 10\% PRP & Yes & Yes & Yes \\
$5 \%$ PRP vs $10 \%$ PRP & No & No & No \\
$5 \%$ PRP vs MTA + 5\% PRP & Yes & Yes & Yes \\
$5 \%$ PRP vs MTA + 10\% PRP & Yes & Yes & Yes \\
$10 \%$ PRP vs MTA + 5\% PRP & No & Yes & Yes \\
$10 \%$ PRP vs MTA + 10\% PRP & No & Yes & Yes \\
MTA + 5\% PRP vs MTA + 10\% PRP & No & Yes & Yes \\
\hline * 0.01 was considered to be statistically significant & &
\end{tabular}

sources in the oral cavity, do exist, there continues to be some lacunae in the standardization of methods of isolation of stem cells and their characterization. ${ }^{27}$ Hence as a baseline for studies on MSCs from other sources in the future, we choose bone marrow MSCs for the present study.

In our study, both concentrations of activated PRP demonstrated that MSC proliferation could be achieved by substituting FCS. The effect of PRP in enhancing the proliferation of MSCs has been demonstrated in other studies as well, irrespective of the preparation being activated $\mathrm{PRP}^{28}$ or non activated $\mathrm{PRP}^{29}$ The proliferative capacity was the same as FCS and did not significantly increase with a higher concentration of PRP. These findings indicate that beyond a certain concentration of PRP, no additional benefits may be attained in terms of expanding the MSCs. On the contrary, a study has reported that increased PRP concentrations (more than $10 \%$ ) may be counterproductive and reduces MSCs' proliferation significantly. ${ }^{30}$

Results from our study also demonstrated that MSCs' proliferation is enhanced by MTA. Proliferation levels in the initial stages were comparable to FCS but in later stages of culture the population of cells was significantly more. Studies on cell lines cultured in direct contact with MTA have shown that the cell proliferation is enhanced. ${ }^{31,32}$ In line with results from our study, MTA has also been reported to promote MSC proliferation in vitro. ${ }^{17}$

On the basis of the positive results observed with PRP and MTA individually, we evaluated MTA combined with two concentrations of PRP. For the first time, we have demonstrated that combining PRP and MTA greatly increases the proliferative effect on MSCs, more so with $10 \%$ concentration. The effect is significantly increased compared to PRP or MTA alone, indicating that the combination has enhanced capacity to induce proliferation of MSCs.

MSCs were induced for osteo/odontogenic differentiation by MTA, PRP, and combined MTA with PRP. Positive staining of the cells with alizarin red indicates the presence of calcium deposition, considered a hallmark of osteo/odontogenic differentiation. 


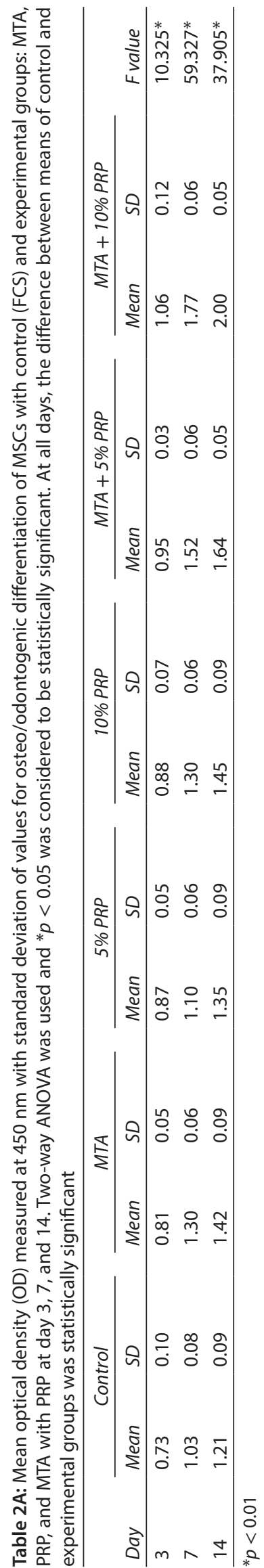

Table 2B: Multiple comparison by Tukey post hoc showed a significant increase in absorbance in MTA with $5 \%$ and $10 \%$ PRP respectively at day 14. No significant difference was observed between control, MTA, 5\% PRP, and 10\% PRP at day 14

Multiple comparsions (MSC osteo/

\begin{tabular}{llll} 
odontogenic differentiation) & Day 3 & Day 7 & Day 14 \\
\hline Control vs MTA & No & Yes & No \\
Control vs 5\% PRP & No & No & No \\
Control vs $10 \%$ PRP & No & Yes & Yes \\
Control vs MTA + 5\% PRP & Yes & Yes & Yes \\
Control vs MTA + 10\% PRP & Yes & Yes & Yes \\
MTA vs 5\% PRP & No & Yes & No \\
MTA vs 10\% PRP & No & No & No \\
MTA vs MTA + 5\% PRP & No & Yes & Yes \\
MTA vs MTA + 10\% PRP & Yes & Yes & Yes \\
$5 \%$ PRP vs $10 \%$ PRP & No & Yes & No \\
$5 \%$ PRP vs MTA + 5\% PRP & No & Yes & Yes \\
$5 \%$ PRP vs MTA + 10\% PRP & Yes & Yes & Yes \\
$10 \%$ PRP vs MTA + 5\% PRP & No & Yes & No \\
$10 \%$ PRP vs MTA + 10\% PRP & No & Yes & Yes \\
MTA + 5\% PRP vs MTA + 10\% PRP & No & Yes & Yes \\
\hline P 20.01 . & &
\end{tabular}

${ }^{*} p<0.01$ was considered to be statistically significant

Similar to the proliferative effect, while MSCs' differentiation was observed with both MTA and PRP, MTA with $10 \%$ PRP showed the maximum differentiation. These results were also verified by means of molar calcium levels wherein the highest degree of calcium deposition was observed when MTA and PRP were combined. MTA is rich in calcium oxide, which is converted to calcium hydroxide on contact with fluid. The calcium hydroxide further dissociates into calcium and hydroxide ions. These calcium ions from MTA have been shown to upregulate BMP-2 in human periodontal ligaments cells, and pulp cells and may form one of the mechanisms by which osteogenic differentiation is promoted. ${ }^{33}$ Other studies have also reported the regulatory effect of MTA on growth factors' release such as FGF-2 and TGF- $\beta$ 1. The interplay of these factors can explain the mineralization by MSCs in the presence of MTA. ${ }^{34}$ Both concentrations of PRP in our study have also demonstrated differentiation of MSCs into osteo/odontogenic lineage. The mineralization rate with PRP was comparable to FCS. In contrast to our study, the rate was reported to be higher in Fetal bovine serum(FBS) followed by $15 \%$ PRP and $10 \% .{ }^{35}$ These contradictions could possibly be due to the variation in PRP preparations and their composition.

The results of the present study demonstrate that while MTA and PRP are individually capable of proliferating cells and inducing osteo/odontogenic differentiation, their combined effect is significantly higher. The underlying mechanisms for the observed effects need further investigation. We hypothesize that the release of calcium ions from MTA form the basis for the release of growth factors in optimum quantities from PRP. Furthermore, the combination plays a role in regulating the actions of various growth factors, which ensures an enhanced effect. The differentiation of both osteoblasts and odontoblasts is characterized by formation of mineral deposits and are indistinguishable without analyzing markers for differentiation. Hence we termed the process as osteo/ odontogenic differentiation. 


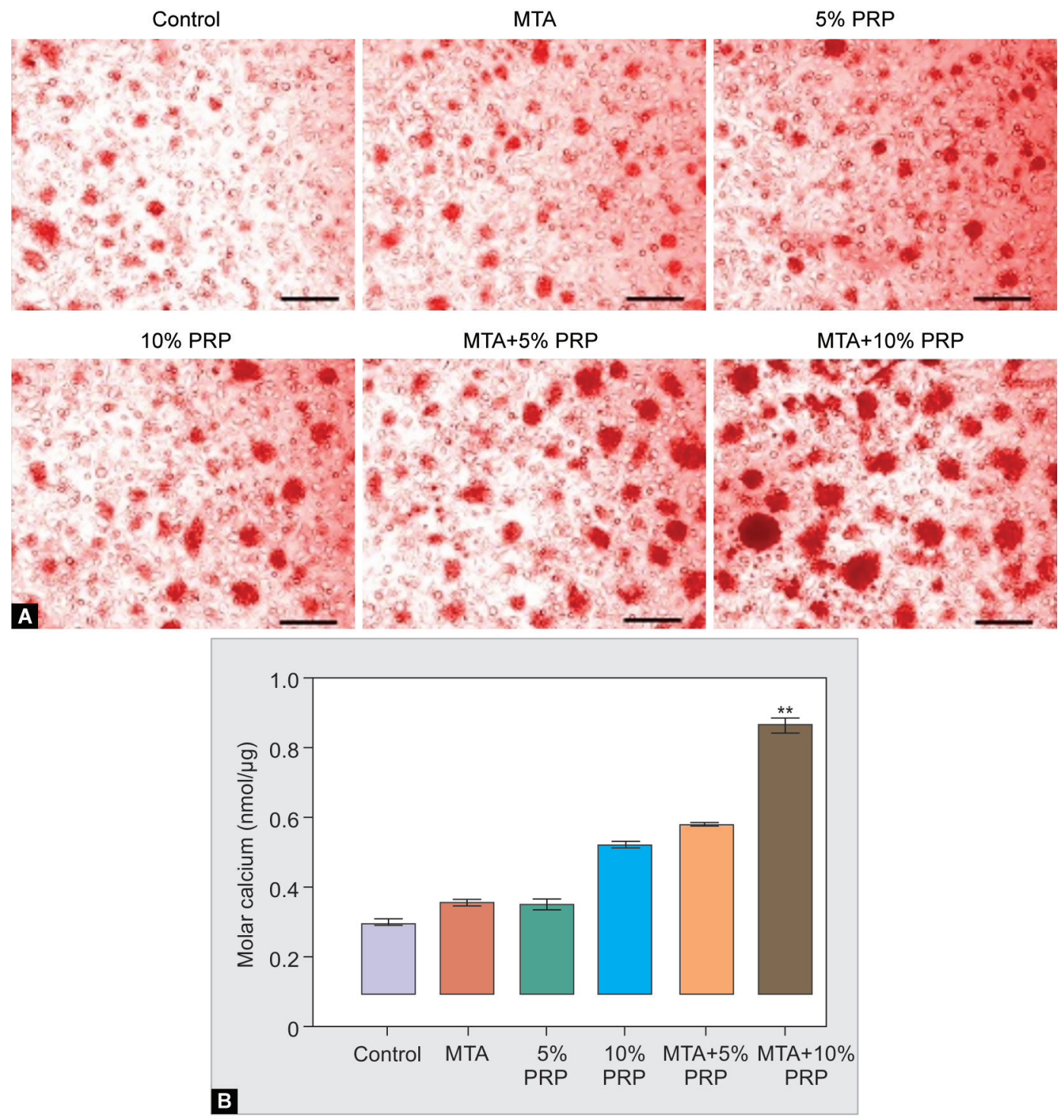

Figs 4A and B: (A) MSCs exhibiting mineralization and nodule formation with 10\% Alizarin red staining in control (10\% FCS) and tested groups at day 14 (magnification: 10X, Scale bar: $50 \mu \mathrm{m}$ ); (B) Calcium deposition (measured by molar calcium ion concentration) at day 14 was the highest in the $10 \%$ PRP + MTA group

\section{CONCLUSION}

The results indicate that both MTA and PRP are individually capable of promoting MSC proliferation in vitro. The combined effect is significantly more than either MTA or PRP and the currently used FCS. Similarly, MSCs are induced for osteo/odontogenic differentiation by both MTA and PRP but together the effect is amplified. Within the limitations of the in vitro environment, we may conclude that MTA in clinical situations does not merely seal the PRP but together they can actually promote cell proliferation and induce mineralization. The mechanisms involved need further investigation as also the phenotype of the cells and mineral deposits formed.

\section{Clinical Significance}

Implications of the results in the clinical environment include a potential for true regeneration of the pulp tissue, rather than revascularization with unpredictable/unfavorable tissue phenotype. Moreover, the combination of PRP and MTA can potentially expand the MSCs to generate adequate numbers for clinical applications, without xenogenic contamination.

\section{Acknowledgment}

We acknowledge the services of Varanasi Satyavani, English Language Instructor, English Language Institute, King Abdulaziz University, Jeddah, Kingdom of Saudi Arabia for the English language revision.

\section{References}

1. Murray PE, Garcia-Godoy F, Hargreaves KM. Regenerative endodontics: a review of current status and a call for action. J Endod 2007;33:377-390. DOI: 10.1016/j.joen.2006.09.013.

2. Thibodeau B, Trope M. Pulp revascularization of a necrotic infected immature permanent tooth: case report and review of the literature. Pediatr Dent 2007;1(29):47-50. 
3. Shah $N$, Logani $A$, Bhaskar $U$, et al. Efficacy of revascularization to induce apexification/apexogenesis in infected, nonvital, immature teeth: a pilot clinical study. J Endod 2008;34:919-925. DOI: 10.1016/ j.joen.2008.05.001.

4. Andreasen JO, Bakland LK. Pulp regeneration after non-infected and infected necrosis, what type of tissue do we want? A review. Dent Traumatol 2012;28:13-18. DOI: 10.1111/j.1600-9657.2011.01057.x.

5. Nosrat A, Homayounfar N, Oloomi K. Drawbacks and unfavorable outcomes of regenerative endodontic treatments of necrotic immature teeth: a literature review and report of a case. J Endod 2012;38:1428-1434. DOI: 10.1016/j.joen.2012.06.025.

6. Schipani E, Kronenberg HM. Adult mesenchymal stem cellsStemBook. Cambridge (MA): Harvard Stem Cell Institute; 2008-2009 Jan 31.

7. Baksh D, Song L, Tuan RS. Adult mesenchymal stem cells: characterization, differentiation, and application in cell and gene therapy. J Cell Mol Med 2004;8:301-316. DOI: 10.1111/j.15824934.2004.tb00320.x.

8. Spees JL, Gregory CA, Singh $\mathrm{H}$, et al. Internalized antigens must be removed to prepare hypoimmunogenic mesenchymal stem cells for cell and gene therapy. Mol Ther 2004;9:747-756. DOI: 10.1016/ j.ymthe.2004.02.012.

9. Hwang YJ, Choi JY. Addition of mesenchymal stem cells to the scaffold of platelet-rich plasma is beneficial for the reduction of the consolidation period in mandibular distraction osteogenesis. J Oral Maxillofac Surg 2010;68:1112-1124. DOI: 10.1016/j.joms.2008.08.038.

10. Astori G, Amati E, Bambi F, et al. Platelet lysate as a substitute for animal serum for the ex vivo expansion of mesenchymal stem/stromal cells: present and future. Stem Cell Res Ther 2016;7:1-8. DOI: 10.1186/ s13287-016-0352-x.

11. Martínez CE, González SA, Palma V, et al. Platelet-Poor and PlateletRich Plasma Stimulate Bone Lineage Differentiation in Periodontal Ligament Stem Cells. J Periodontol 2016;87:e18-e26. DOI: 10.1902/ jop.2015.150360.

12. Martin G, Ricucci D, Gibbs JL, et al. Histological findings of revascularized/revitalized immature permanent molar with apical periodontitis using platelet-rich plasma. J Endod 2013;39:138-144. DOI: 10.1016/j.joen.2012.09.015.

13. Bezgin T, Yılmaz AD, Celik BN, et al. Concentrated platelet-rich plasma used in root canal revascularization: 2 case reports. Int Endod J 2014;1:41-49. DOI: 10.1111/iej.12144.

14. Torabinejad $\mathrm{M}$, Faras $\mathrm{H}$. A clinical and histological report of a tooth with an open apex treated with regenerative endodontics using platelet-rich plasma. J Endod 2012;38:864-868. DOI: 10.1016/ j.joen.2012.03.006.

15. Stefopoulos S, Tsatsas DV, Kerezoudis NP, et al. Comparative in vitro study of the sealing efficiency of white vs grey ProRoot mineral trioxide aggregate formulas as apical barriers. Dent Traumatol 2008;24:207-213. DOI: 10.1111/j.1600-9657.2007.00516.x.

16. Mozayeni MA, Milani AS, Marvasti LA. Cytotoxicity of calcium enriched mixture cement compared with mineral trioxide aggregate and intermediate restorative material. Aust Dent J 2012;38:70-75. DOI: 10.1111/j.1747-4477.2010.00269.x.

17. D'Antò V, Di Caprio MP, Ametrano G, et al. Effect of mineral trioxide aggregate on mesenchymal stem cells. J Endod 2010;36:1839-1843. DOI: 10.1016/j.joen.2010.08.010.

18. Bausset O, Giraudo L, Veran J, et al. Formulation and storage of platelet-rich plasma homemade product. Biores Open Access 2012;1:115-123. DOI: 10.1089/biores.2012.0225.

19. Amit V, Vishwakarma SK, Vanka GS, et al. In vitro proliferation of MSCs using mineral trioxide aggregate: a most recent material for in situ stem cells mobilisation. Int J Adv Res 2014;2:561-567.
20. Qian $Y$, Han $Q$, Chen W, et al. Platelet-rich plasma derived growth factors contribute to stem cell differentiation in musculoskeletal regeneration. Front Chem 2017;5:89. DOI: 10.3389/fchem.2017. 00089.

21. Dhurat R, Sukesh MS. Principles and methods of preparation of platelet-rich plasma: a review and author's perspective. J Cutan Aesthet Surg 2014;7(4):189. DOI: 10.4103/0974-2077.150734.

22. Mazzucco L, Balbo V, Cattana E, et al. Not every PRP-gel is born equal. Evaluation of growth factor availability for tissues through four PRP-gel preparations: Fibrinet, RegenPRP-Kit, Plateltex and one manual procedure. Vox Sang 2009;97:110-118. DOI: 10.1111/j.14230410.2009.01188.x.

23. Dohan Ehrenfest DM, Rasmusson L, Albrektsson T. Classification of platelet concentrates: from pure platelet-rich plasma (P-PRP) to leukocyte- and platelet-rich fibrin (L-PRF). Trends Biotechnol 2009;27:158-167. DOI: 10.1016/j.tibtech.2008.11.009.

24. Perut F, Filardo G, Mariani E. Preparation method and growth factor content of platelet concentrate influence the osteogenic differentiation of bone marrow stromal cells. Cytotherapy 2013;15:830-839. DOI: 10.1016/j.jcyt.2013.01.220.

25. Hass R, Kasper C, Böhm S, et al. Different populations and sources of human mesenchymal stem cells (MSC): A comparison of adult and neonatal tissue-derived MSC. Cell Commun Signal 2011;9:12. DOI: 10.1186/1478-811X-9-12.

26. Nombela-Arrieta C, Ritz J, Silberstein LE. The elusive nature and function of mesenchymal stem cells. Nat Rev Mol Cell Biol 2011;12:126. DOI: 10.1038/nrm3049.

27. Avinash K, Malaippan S, Dooraiswamy JN. Methods of isolation and characterization of stem cells from different regions of oral cavity using markers: a systematic review. Int J Stem Cells 2017;10:12. DOI: 10.15283/ijsc17010.

28. Vogl M, Fischer J, Jäger $M$, et al. Can thrombin-activated platelet releasate compensate the age-induced decrease in cell proliferation of MSC? J Orthop Res 2013;11:1786-1795. DOI: 10.1002/jor. 22433.

29. Mishra A, Tummala $P$, et al. Buffered platelet-rich plasma enhances mesenchymal stem cell proliferation and chondrogenic differentiation. Tissue Eng Part C Methods 2009;15:431-435. DOI: 10.1089/ten.tec.2008.0534.

30. Cho HS, Song IH, Park SY, et al. Individual variation in growth factor concentrations in platelet-rich plasma and its influence on human mesenchymal stem cells. Korean J Lab Med 2011;31:212-218. DOI: 10.3343/kjlm.2011.31.3.212.

31. Chen $\mathrm{CL}$, Huang TH, Ding SJ, et al. Comparison of calcium and silicate cement and mineral trioxide aggregate biologic effects and bone markers expression in MG63 cells. J Endod 2009;35:682-685. DOl: 10.1016/j.joen.2009.02.002.

32. Takita T, Hayashi M, Takeichi O, et al. Effect of mineral trioxide aggregate on proliferation of cultured human dental pulp cells. Int Endod J 2006;39:415-422. DOI: 10.1111/j.1365-2591.2006. 01097.x.

33. Maeda $\mathrm{H}$, Nakano $\mathrm{T}$, Tomokiyo $\mathrm{A}$, et al. Mineral trioxide aggregate induces bone morphogenetic protein-2 expression and calcification in human periodontal ligament cells. J Endod 2010;36:647-652. DOI: 10.1016/j.joen.2009.12.024.

34. Kwon JY, Lim SS, Baek SH, et al. The effect of mineral trioxide aggregate on the production of growth factors and cytokine by human periodontal ligament fibroblasts. J Korean Acad Conserv Dent 2007;32:191-197. DOI: 10.5395/JKACD.2007.32.3.191.

35. Tavakolinejad S, Khosravi M, Mashkani B, et al. The effect of human platelet-rich plasma on adipose-derived stem cell proliferation and osteogenic differentiation. Iran Biomed J 2014;18:151. 